\title{
Plantes, peuplements cultivés et territoires
}

\section{Crops, planting and farmland}

Oléagineux, Corps Gras, Lipides. Volume 7, Numéro 6, 494-8, Novembre - Décembre 2000, Dossier : "Agriculture, recherche et territoire"

Auteur(s) : Gilles Lemaire, Département Environnement et agronomie, Inra, route de Saintes, 86600 Lusignan, France.

Résumé : Les territoires agricoles sont structurés en fonction des divers objectifs qui peuvent être assignés à la production agricole. Ces objectifs ont trait soit à l'économie des exploitations agricoles et des filières de production, soit à la maîtrise des impacts sur l'environnement, soit à la gestion des ressources naturelles, soit à la gestion de la qualité des produits, soit plus généralement à la gestion des paysages et au développement rural. À chacun de ces objectifs correspondent des entités territoriales fonctionnelles différentes qui imposent des critères d'évaluations spécifiques aux parcelles cultivées qui y sont inclues. Ceci nécessite donc une analyse multicritères du fonctionnement de la parcelle cultivée qui ouvre largement la gamme de contraintes du milieu à prendre en compte. Dans ce contexte, l'évaluation du progrès génétique mis à disposition de l'agriculture devra se faire également sur une base fonctionnelle plus large. L'analyse du fonctionnement des plantes individuelles au sein des peuplements cultivés devient alors indispensable pour expliciter les régulations génétiques mises en œuvre et proposer une véritable analyse fonctionnelle des interactions " génotypes-milieu - itinéraires techniques ».

Mots-clés : systèmes de culture, interactions " génotype-milieu ", modélisation, environnement, entités spatiales fonctionnelles, peuplement végétal, territoire.

Summary : Agricultural territories are structured in relation with the different goals assigned to agriculture. These goals are related to (i) economy of farms and integrated chains of production, (ii) environmental impacts, (iii) natural resource preservation, (iv) quality of products, (v) landscape management and rural development. Each of these objectives need to be analysed within different functional spatial entities each of them imposing specific evaluation criteria for crops at field scale level. Thus it is necessary to develop a multi-criteria analysis of crop functioning taking into account a wider range of environmental constraints. In such a new context, the evaluation of new cultivars offered to farmers should be based also on a large multi-functional analysis. Models based on an explicit individual plant functioning within plant populations become necessary for interpreting the "genotype by environment by crop management" interactions in a functional way leading to the identification of regulations of genes expressions.

Keywords : cropping systems, genotype by environment interactions, modelling, environment, functional spatial entities, crops, landscape.

\section{ARTICLE}


Les paysages de nos régions, tels que nous les avons connus et tels que nous les voyons aujourd'hui, sont profondément marqués par la nature des espèces qui sont cultivées et par les modes de culture qui sont pratiqués. Mais, au-delà de cet aspect le plus visible, les relations entre les plantes cultivées et les territoires s'exercent à de multiples niveaux. Ainsi, les gains de productivité qui ont été obtenus sur la plupart des grandes espèces cultivées sont le résultat d'un ajustement constant entre le progrès génétique et une modification des contraintes biotiques et abiotiques du milieu, à l'échelle du champ cultivé, par les pratiques culturales, et c'est le résultat de ces ajustements qui a contribué à modifier profondément la physionomie des territoires ruraux.

Il y a donc un premier niveau d'analyse des relations entre les espèces cultivées et les territoires qui concerne l'étude explicite des termes de passage entre l'échelle d'organisation de l'individu biologique qui est la plante, support de l'information génétique et de ses interactions avec le milieu, et l'échelle d'organisation du peuplement végétal et du champ cultivé qui représente l'entité sur laquelle s'exercent et s'évaluent les pratiques agronomiques. L'ambition de ce premier niveau d'analyse est de montrer comment progrès génétique et pratiques culturales doivent interagir pour optimiser le fonctionnement de la parcelle cultivée dans un territoire donné.

Un deuxième niveau d'analyse est nécessaire pour définir, à l'échelle d'un territoire, les critères pertinents d'optimisation de la conduite des champs cultivés qui y sont inclus. Ceci revient à dire qu'une parcelle cultivée ne peut pas fournir " en soi " de critères d'optimisation de son propre fonctionnement. Ces critères ne trouvent leur pertinence que dans le cadre de niveaux d'organisations plus englobants : l'exploitation agricole pour certains critères socio-économiques, l'entité spatiale fonctionnelle du point de vue de l'environnement (bassins versants, zones vulnérables, habitats d'espèces protégées, etc.) du point de vue des filières de production (zones de collecte, appellations d'origine, etc.), ou du point de vue réglementaire et socio-politique (qualification, certification, développement rural, etc.). L'optimisation de toutes ces fonctions au sein des différentes entités territoriales auxquelles une même parcelle peut être rattachée doit définir le cahier des charges à partir duquel les critères d'optimisation du fonctionnement de cette parcelle cultivée devront être définis. En d'autres termes, les territoires ruraux ne doivent pas être considérés comme une juxtaposition de parcelles cultivées dont on assurerait pour chacune d'elles un fonctionnement dit "optimalisé " en agissant sur les interactions entre génotypes, milieux et pratiques agricoles, mais comme un système organisé définissant un champ spatialisé de contraintes de natures différentes. Comme dans tout système organisé et hiérarchisé, l'optimisation du tout n'est pas obtenue par le fonctionnement optimum des parties.

Pendant longtemps, le critère essentiel d'évaluation agronomique des champs cultivés a été celui de la " marge brute ", toute augmentation de celle-ci étant considérée comme a priori bénéfique pour chaque exploitation agricole, quel que soit le territoire considéré. Ainsi, les critères du progrès génétique et la recherche des optimums de pratiques ont été guidés par les rapports de prix entre intrants et produits récoltés. Plus récemment, les préoccupations de durabilité des systèmes de production agricole ont imposé une prise en compte plus explicite des interactions entre les parcelles cultivées et des entités territoriales fonctionnelles plus vastes. Ceci implique que soient reconsidérés en partie les concepts, approches et méthodes qui permettent d'analyser l'optimisation des interactions entre progrès génétique et conduite du champ cultivé afin de prendre en compte simultanément des critères relatifs à la rentabilité économique des systèmes de production, aux exigences de qualité et de traçabilité des produits, et aux impacts environnementaux positifs et 
négatifs... qui traduisent la diversité des objectifs de l'activité agricole à l'échelle d'un territoire.

\section{Champs cultivés et contraintes à l'échelle du territoire}

La nécessité d'une prise en compte de la multi-fonctionnalité de l'activité agricole implique l'abandon d'une évaluation mono-critère $d u$ fonctionnement du champ cultivé dans le cadre d'une performance économique de l'exploitation agricole considérée comme " isolée " ou du moins " extraite " des différents territoires dans lesquels elle est insérée. II importe alors de déterminer de manière beaucoup plus explicite, à l'échelle d'un territoire, le cadre spatial dans lequel les différentes contraintes s'exercent sur la production agricole.

Il s'agit d'une démarche essentiellement " holistique » qui doit considérer les territoires comme des entités dont les fonctions sont distribuées et hiérarchisées dans l'espace. La distribution et la structuration spatiale de ces fonctions sont bien entendu différentes pour chacune d'entre elles. Ainsi, le fonctionnement d'un territoire vis-à-vis de fonctions environnementales comme la gestion quantitative et qualitative des ressources en eau est déterminé par sa structure hydrographique et hydrogéologique ainsi que par celle de la couverture pédologique qui déterminent à la fois les contraintes du milieu qui s'appliquent aux parcelles cultivées ainsi que les cahiers des charges qui peuvent être localement imposés à leur fonctionnement. Par exemple, le fonctionnement hydrique d'une parcelle sera analysé et évalué différemment selon qu'elle est située en haut de coteaux en zone fortement contributive pour le ruissellement ou en bas de pente en zone hydromorphe. De même, l'impact des modes de conduite d'une parcelle sur la gestion qualitative et quantitative de l'eau à l'échelle d'un bassin versant sera différent selon le niveau de réserve utile du sol. Ainsi, les décisions qui devront être prises sur la nature des cultures à répartir sur un territoire et sur les itinéraires techniques qui devront être localement mis en œuvre devront être évaluées en fonction de cette différentiation spatiale caractéristique du territoire considéré. De la même manière, la gestion de la qualité des produits récoltés à l'échelle d'un territoire implique une analyse de la structuration spatiale des contraintes physiques du milieu (sol et climat) qui peuvent induire une différenciation qualitative identifiable des produits en interaction avec les pratiques (effets terroirs) ainsi qu'une prise en compte de l'organisation territoriale des agents économiques qui assurent la collecte et la mise en lot des récoltes afin de savoir comment valoriser au mieux les différences qualitatives potentiellement existantes (voir l'article de Marianne Le Bail dans ce même numéro).

De telles analyses de la structuration spatiale d'un territoire doivent être entreprises pour différents critères ayant trait à la fois à des fonctions environnementales (qualité des eaux et de l'air, conservation et qualité des sols, gestion de la biodiversité faunistique et floristique), à des fonctions économiques (rentabilité des exploitations agricoles et des filières de production, identification, et valorisation de la qualité des produits agricoles, développement rural) et à des fonctions sociétales (acceptabilité des modes de production, certification des pratiques et traçabilité des produits, gestion patrimoniale des paysages, etc.). Toutes ces fonctions n'ont de sens qu'appliquées à des portions d'espace qui sont d'une dimension supérieure à celle du champs cultivé. Elles imposent donc à ce dernier un cadre de contraintes multiples au sein duquel devront être élaborés les critères qui permettent d'en évaluer le fonctionnement. C'est à ce titre que la durabilité des systèmes de culture pourra être évaluée. Ainsi, un système de culture défini par la succession des cultures sur un ensemble de parcelles et par les itinéraires techniques appliqués à ces cultures et aux intercultures [1] pourra être considéré comme " durable » sur une portion d'un territoire alors qu'il pourra être 
considéré comme « non durable » sur une autre portion de ce même territoire.

Cette vision " intégrée " de la durabilité des systèmes qui implique la prééminence du fonctionnement des territoires sur les champs cultivés a des conséquences importantes sur l'étude du fonctionnement des cultures. En effet, l'évaluation du fonctionnement des cultures était jusqu'à présent entièrement dominée par le concept de " rendement potentiel », le diagnostic agronomique mesurant l'écart entre le " rendement réalisé » et le rendement potentiel calculé en fonction des contraintes permanentes du milieu local et du potentiel génétique. Ce critère d'évaluation unique était alors utilisé indépendamment du contexte territorial et il a servi d'étalon à tout le développement agricole. Seuls ont gardé une approche plus systémique les systèmes de production fortement inféodés à un territoire comme les systèmes d'élevage en zones herbagères. II est intéressant de noter que, pour ces systèmes, la notion même de " rendement " n'était pas opératoire puisque, dans un système fourrager, c'est l'adéquation spatio-temporelle de la production d'herbe à un cahier des charges fixé par un troupeau qui s'impose et qui représente déjà, à l'échelle de la sole fourragère, une démarche multicritère de type territoriale [2].

Pour illustrer ce changement d'optique dans l'évaluation du fonctionnement du champ cultivé, nous allons en analyser les conséquences dans le cadre d'un objectif de gestion territoriale des ressources quantitative et qualitative en eau. $\mathrm{Si}$, pour un territoire donné, on imagine une ressource en eau quantitativement définie, de type " nappe fermée ", dont on doit assurer le renouvellement, la problématique territoriale consiste à savoir quelle est la part de la ressource annuelle disponible pour la ré-alimentation de la nappe (pluies-évapotranspiration réelle) qui peut être prélevée pour l'irrigation sans que le renouvellement de la ressource soit affecté. Cette quantité d'eau disponible permettra de définir la surface maximum irrigable à l'échelle du territoire. Dans ce cadre, la problématique sera essentiellement celle de la répartition des cultures irrigués et non irriguées à l'échelle du territoire, tandis qu'une politique de restriction ou de contrôle de l'irrigation à l'échelle de la parcelle est peu pertinente, une irrigation excédentaire n'ayant aucun effet sur le bilan de la nappe à moyen terme et n'ayant comme inconvénient qu'une diminution de la rentabilité économique de l'irrigation pour la parcelle considérée. En revanche, dans un tel système, une mauvaise estimation du poste " évapotranspiration " à l'échelle du territoire, du fait d'une mauvaise analyse de la répartition spatiale des cultures en fonction des réserves hydriques des sols, peut avoir des conséquences importantes en conduisant les décideurs à surestimer les volumes d'eau qui peuvent être prélevés pour l'irrigation, ce qui conduirait à une gestion non durable de la ressource. Dans ce cas, l'analyse agronomique du fonctionnement des champs cultivés doit porter en priorité non sur les "parcelles irriguées " mais sur le fonctionnement des cultures " non irriguées " dont le comportement spatialisé conditionne effectivement le volume d'eau qui est susceptible de retourner à la nappe et donc, in fine, les volumes qui peuvent en être soustraits pour l'irrigation. En revanche, dans un territoire où la ressource en eau est de type " ouvert " comme dans le cas d'une rivière, le facteur qui limite l'utilisation de la ressource est le débit d'utilisation en période d'étiage. Dans ce cadre de contrainte, il est clair que toute irrigation excédentaire du fait d'une mauvaise gestion de certaines parcelles irriguées contribue à limiter la ressource disponible pour les autres parcelles. Une problématique de restriction des apports en fonction de la dynamique de besoin des cultures doit alors être recherchée à travers une analyse du fonctionnement des cultures irriguées et traduite en termes de pilotage de l'irrigation. On voit à travers ces deux exemples que la problématique de l'évaluation du fonctionnement des champs cultivés par rapport à la gestion quantitative des ressources en eau à l'échelle d'un territoire dépend grandement du contexte territorial. Or, face à ces 
problématiques diverses, les écophysiologistes qui analysent le fonctionnement hydrique des cultures ont eu tendance à se contenter d'un critère d'évaluation " unique " qui est l'efficience de l'eau. Si ce concept demeure pertinent du point de vue écologique pour classer entre elles des espèces, il apparaît peu opératoire dans une optique de gestion des ressources en eau. L'efficience de l'eau mesure la capacité d'une espèce à produire de la matière sèche (ou mieux de la biomasse récoltée) par unité de volume d'eau consommée. Or, une augmentation d'efficience de l'eau peut être obtenue soit par une augmentation de la capacité de production de la culture, soit par une diminution de sa consommation en eau, ce qui n'est pas du tout équivalent pour le gestionnaire d'une ressource en eau. Savoir qu'une augmentation de l'efficience de l'eau est possible est certes très intéressant mais, lorsque celle-ci est accompagnée d'une augmentation substantielle des volumes d'eau consommés sur un territoire, cela en limite déjà beaucoup la portée. C'est le cas du maïs qui, du fait de son métabolisme en $\mathrm{C}_{4}$, possède une efficience de l'eau supérieure à celle d'autres cultures comme le blé ou le tournesol. Cependant, cette forte efficience, gage d'une bonne rentabilité économique de l'irrigation de cette espèce à l'échelle de la parcelle, ne s'extériorise que grâce à des consommations importantes à la fois en volumes et en débits du fait de la coïncidence des besoins de la culture avec les périodes de très forte demande climatique. Ainsi, ce qui peut être considéré comme " optimum » à l'échelle de la parcelle peut devenir « contraignant » à l'échelle d'un territoire. L'irrigation d'une espèce comme le blé, ou le tournesol, moins efficiente que celle du maïs à l'échelle de la parcelle pourrait s'avérer cependant pertinente dans un cadre territorial. À cet égard, l'intérêt d'une espèce comme le sorgho, qui possède une efficience de l'eau comparable à celle du maïs mais qui nécessite des volumes d'eau moindres du fait de son niveau de production plus réduit, pourrait être étudié. Une analyse purement comparative des performances des cultures en termes de rendement et d'efficience de l'eau à l'échelle de la parcelle cultivée a tendance aujourd'hui à condamner irrémédiablement toute autre culture que le maïs sans que les analyses de changement d'échelle " parcelle-territoire " aient fourni un cadre cohérent d'évaluation qui devrait aboutir à une analyse de la complémentarité relative entre les différentes cultures plutôt qu'à un classement hiérarchique en valeur absolue.

Lorsque l'on aborde le problème de la gestion qualitative de l'eau à l'échelle territoriale, il s'agit de limiter la concentration en nitrate des eaux qui drainent dans les sols et ré-alimentent les aquifères. Or, ce problème a souvent été traduit au niveau de la parcelle cultivée par une problématique de réduction des pertes d'azote et une approche de type " bilan ». Une même quantité d'azote perdue par lixiviation à l'échelle d'un territoire n'a pas la même conséquence en termes de qualité des eaux souterraines selon la hauteur de la lame d'eau drainante qui ré-alimente les nappes. Si l'on veut traduire cette contrainte en termes de critère de fonctionnement du champ cultivé, il faut alors combiner des indicateurs de fonctionnement hydrique de la culture avec des indicateurs de prélèvement d'azote. Ainsi, si l'on exprime les capacités de prélèvement de $\mathrm{N}$ d'une culture en fonction de ses capacités de prélèvements d'eau ( $\left.\mathrm{kg} \mathrm{N} / \mathrm{mm} \mathrm{H}_{2} \mathrm{O}\right)$, on détermine alors une " concentration équivalente " de la lame d'eau évapotranspirée par la culture [3]. Une variation de cette " concentration équivalente " de l'eau consommée à l'échelle d'un cycle de culture doit se traduire, toutes conditions égales, par une variation inverse de la concentration moyenne de la lame d'eau qui drainera pendant la période hivernale. Un tel indice, aisément calculable à partir des modèles de fonctionnement de culture, peut permettre un classement relatif des cultures et systèmes de culture vis-à-vis des risques de pollution nitrique. Ce ne sont pas les cultures qui possèdent les plus fortes capacités de prélèvement d'azote qui permettent de minimiser les risques, 
mais bien celles qui optimisent ce prélèvement d'azote tout en prélevant le minimum d'eau. Ainsi, le sorgho s'avère être une espèce plus " épuratrice " que le maïs en conditions non irriguées [3]. Dans un sol donné et pour une culture donnée, le rapport de prélèvement $\mathrm{N} / \mathrm{H}_{2} \mathrm{O}$ augmente lorsque que le prélèvement d'eau du sol par la culture s'effectue majoritairement dans les horizons superficiels qui sont enrichis en azote. L'irrigation est un moyen de concentrer le fonctionnement racinaire dans les horizons les plus superficiels riches en azote et, si cette pratique n'est pas accompagnée de niveaux de fertilisation excédentaires, elle peut contribuer à limiter la concentration en nitrate de la lame d'eau drainante, d'une part, grâce à une augmentation du volume de cette dernière et, d'autre part, du fait d'un prélèvement plus efficace de l'azote. En revanche, en culture sèche, le prélèvement d'azote des horizons superficiels est limité par le manque d'eau alors que la culture continue à s'alimenter en eau dans les horizons profonds pauvres en azote, ce qui a tendance à diminuer le rapport $\mathrm{N} / \mathrm{H}_{2} \mathrm{O}$ prélevée. L'architecture racinaire des espèces cultivées apparaît donc comme une caractéristique déterminante. Un enracinement dense mais superficiel limitant le volume d'eau du sol extractible par la culture mais optimisant le prélèvement de l'azote devrait conduire à une optimisation du rapport $\mathrm{N} / \mathrm{H}_{2} \mathrm{O}$ prélevée et ainsi diminuer les risques à l'échelle de la parcelle cultivée. On voit aisément que ce critère favorable pour diminuer le risque de "pollution nitrique " pourrait être associé à un critère défavorable pour la productivité puisqu'une limitation du volume d'eau extractible par la culture devrait augmenter le risque de baisse de rendement en situation de sécheresse. II s'agit donc d'un problème de gestion de compromis entre deux risques qui dépendra grandement de la hiérarchisation qui sera faite entre ceux-ci à l'intérieur d'un territoire.

\section{Plantes et contraintes à l'échelle du peuplement végétal}

Nous avons analysé ci-dessus à travers quelques exemples comment des contraintes définies à l'échelle d'un territoire pouvaient déterminer le cahier des charges du fonctionnement des champs cultivés et se traduire par des critères multiples d'évaluation. Ainsi, le choix des espèces cultivées et leur répartition spatiale au sein d'un territoire doivent se raisonner en fonction des aptitudes de ces espèces à satisfaire ces critères d'évaluation dans les différentes conditions de contraintes environnementales spatialement réparties dans ce territoire. II importe donc de traduire ces critères en caractéristiques spécifiques des différentes espèces cultivées. Bien plus, pour une même espèce cultivée, le choix des variétés adaptées aux conditions de milieux et aux itinéraires techniques imposés par le fonctionnement du territoire devra lui aussi se faire sur la base d'une analyse des interactions " génotypes - milieu - itinéraires techniques » (voir l'article de Maryse Brancourt-Hulmel dans ce même numéro). Au-delà de ces critères de choix du matériel végétal existant, l'orientation stratégique du progrès génétique par la sélection végétale doit prendre en compte la multiplicité des critères d'évaluation que nous avons évoqués ci-dessus pour déterminer les idéotypes de plantes qui seront constitutifs des peuplements végétaux. Cette évaluation multicritère des aptitudes du matériel végétal se heurte à des incompatibilités ou du moins à des contradictions entre critères qui ne pourront être résolues que grâce à une plus grande diversification et à une " spécialisation " plus affirmées des types variétaux en fonction, d'une part, de l'élargissement de la gamme de contraintes biotiques et abiotiques qu'il y aura lieu de prendre en compte et, d'autre part, de la diversité dans la hiérarchie des objectifs visés (niveaux et coûts de production, environnement, qualité des produits).

Les outils d'évaluation du fonctionnement des peuplements cultivés sont actuellement de deux 
types. Soit il s'agit de modèles de simulation de culture tels que STICS [4] ou CERES [5] qui permettent de prédire un certain nombre de variables de la culture (niveau de production, niveau de consommation en eau et en azote, etc.) ou du milieu (bilan d'eau, bilan de $\mathrm{N}$ et de $\mathrm{C}$, etc.) à partir de variables d'entrée ayant trait au milieu (contraintes pédo-climatiques) ou relatives aux modes de conduite. Soit il s'agit d'outils de diagnostics permettant, à partir d'une mesure, de calculer un indicateur de fonctionnement de la culture relatif à une variable d'état du champ cultivé comme l'indice de nutrition azotée [6]. En réalité, ces deux types d'outils sont étroitement complémentaires et interactifs. Leur origine est d'ailleurs commune. Elle provient d'une analyse globale du fonctionnement des cultures dans laquelle le peuplement végétal est considéré comme une interface " physique " qui régule les échanges de masse et d'énergie entre le sol et l'atmosphère. Ces fonctions d'échange sont paramétrées pour rendre compte des régulations biologiques sous-jacentes relatives à des fonctions telles que la photosynthèse, la transpiration, l'absorption minérale, la morphogenèse... et elles sont combinées dans le cadre d'une approche de type " captationconversion » des ressources du milieu. Mais les algorithmes et les coefficients d'ajustements qui sont utilisés ne sont pas toujours explicités au niveau du fonctionnement de l'entité biologique, c'est-àdire la plante individuelle qui reste la base incontournable de l'analyse de l'expression du génome. Ainsi, ces modèles de fonctionnement permettent d'analyser avec beaucoup d'efficacité le fonctionnement des peuplements cultivés en simulant les flux aux bornes du système et sont donc des outils indispensables pour en évaluer le fonctionnement global du double point de vue " production " et " environnement ". La mise en œuvre de ces outils est tout à fait pertinente dans le cadre d'une évaluation du fonctionnement des champs cultivés à l'échelle d'un territoire car ils permettent, en outre, un couplage avec une analyse spatialisée des contraintes par l'intermédiaire des systèmes d'informations géographiques.

Cependant, au-delà de ces aspects opérationnels qui doivent continuer à être développés, il est indispensable de développer une deuxième génération d'outils d'analyse du fonctionnement des peuplements cultivés qui puissent identifier de manière plus explicite les fonctions biologiques telles qu'elles sont effectivement régulées et contrôlées génétiquement à l'échelle de l'individu biologique qui est la plante. II convient alors de ne plus considérer le peuplement végétal comme une interface d'échange entre compartiments du milieu, mais bien comme une collection de plantes individuelles qui interagissent entre elles et dont le fonctionnement résultant détermine les " propriétés émergentes " du système. Ceci revient donc : - à prendre en compte les champs de contraintes qui s'exercent sur les plantes individuelles au sein des peuplements du fait des interférences avec les plantes voisines;

- à décrire et analyser les interactions entre plantes au sein des peuplements végétaux, ce qui est classiquement appelé " compétition », tant en termes de partage des ressources, qu'en termes de « signaux " ;

- à expliciter les régulations génétiques mises en jeu et exprimées au niveau des plantes individuelles sous l'effet de la compétition ;

- à établir les termes de passage du niveau d'organisation « plante » à celui de " population de plante 
». Ce type d'analyse nous paraît indispensable pour aboutir, avec les outils de la génomique fonctionnelle, à une identification des régulations génétiques qui sont réellement mises en œuvre à l'échelle des plantes individuelles et qui déterminent les réponses adaptatives attendues à l'échelle des peuplements végétaux.

L'analyse de la compétition entre plantes individuelles au sein des peuplement végétaux n'a été réellement entreprise que pour des peuplements plurispécifiques. Or, même dans ces situations, les approches ont été essentiellement de type statistique et n'ont donc pas abouti à une identification des régulations génétiques mises en jeu. Même dans le cas des peuplements monospécifiques, il importe de rendre plus explicites les termes de passage du niveau d'organisation de la plante à celui du peuplement. En effet, la plupart des approches globales du fonctionnement des peuplements se heurtent à la difficulté de représentation de la répartition des assimilats entre les différents organes d'une plante. Or, une telle analyse est indispensable pour une étude de la dynamique de mise en place et de la croissance des organes récoltés (graines, fruits, tubercules, etc.) ainsi que de leur composition. Une approche de type purement compartimentale n'est plus opérante à partir du moment où l'architecture des plantes qui détermine les relations topologiques et les échanges trophiques entre ces différents organes au sein d'une plante est elle-même dépendante des relations de compétition existant entre plantes au sein d'un peuplement. Cela signifie que l'approche de type " plante moyenne " rencontre des limites qui ne permettent pas d'explorer toute la gamme de conditions dans lesquelles s'exercent les compétitions entre plantes. L'analyse des composantes du rendement, outil du diagnostic agronomique, montre ainsi ses limites puisqu'elle ne permet pas d'expliciter les compensations existant entre les différentes composantes (par exemple entre nombre de plantes, taille des plantes et nombre de talles) autrement que par une relation statistique appliquée à une plante moyenne qui représente une entité biologique virtuelle. II est donc indispensable de passer d'une approche purement compartimentale de régulation des flux, qui est la base de la plupart des modèles de simulation de culture utilisés aujourd'hui, à une approche de type " structure-fonction " qui permettra de fournir un cadre pour l'analyse de l'expression des régulations génétiques à l'échelle d'une population d'individus.

L'évaluation du progrès génétique à l'échelle du champ cultivé et l'identification des voies d'amélioration possibles de celui-ci représentent les deux facettes d'un même problème qui est posé en commun à un ensemble de disciplines scientifiques telles que la biologie végétale cellulaire et moléculaire, la génétique et l'écophysiologie qui doivent forger ensemble les outils d'analyse et d'évaluation des peuplements végétaux qui puissent être pertinents à la fois vis-à-vis des fonctions attendues de ces peuplements dans un territoire donné et vis-à-vis de l'identification des déterminants génétiques de ces fonctions. II s'agit donc de développer une gamme d'outils différents s'appliquant à des niveaux d'organisation différents depuis l'organe, la plante individuelle, la population de plantes en compétition et le champ cultivé, en veillant à assurer la compatibilité et la cohérence entre ces outils qui devront pouvoir être " interfacés ». II ne s'agit donc pas de remplacer une gamme d'outils par une autre mais bien de compléter la gamme existante. L'analyse du fonctionnement des peuplements végétaux apparaît donc être le carrefour entre une démarche de type " descendante ", qui assimile le peuplement végétal à un système régi par les lois de la thermodynamique, et une démarche " ascendante ", qui considère le peuplement végétal comme un ensemble structuré de gènes qui régulent des fonctions physiologiques.

De cette confrontation entre le champ des possibles (génome) et le champ des contraintes (milieu 
physique) émergent les gammes d'adaptation du fonctionnement des peuplements végétaux qui peuvent être envisagées pour le progrès génétique.

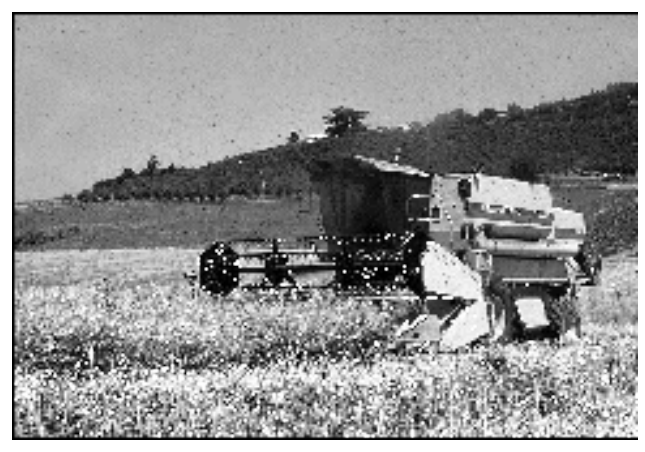

(Photo J. Raimbault, Cetiom)

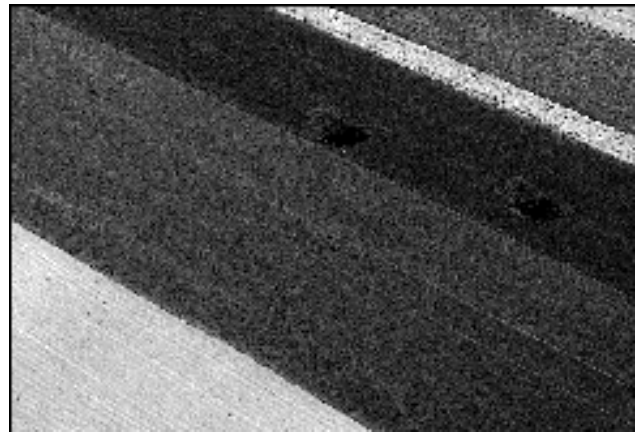

(Photo C. Slagmulder, Inra)

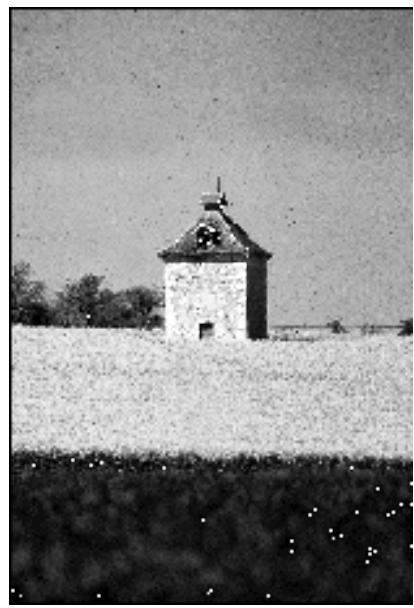

(Photo G. Cattiau, Inra)

\section{CONCLUSION}

La diversification des objectifs qui sont poursuivis par l'activité agricole, afin de s'insérer de manière plus efficace dans une stratégie de développement territoriale, impose d'évaluer le fonctionnement des champs cultivés selon des critères à la fois plus précis et multiples. Les possibilités technologiques nouvelles permettant une modification à la fois plus rapide et plus profonde du génome des espèces cultivées laissent entrevoir une accélération du progrès génétique. Il est donc important, si l'on veut que ce progrès soit orienté vers les problématiques qui se posent réellement à l'échelle des territoires, d'une part, d'expliciter les diverses fonctions attendues au niveau des champs cultivés au sein des territoires, 
d'autre part, de traduire ces fonctions en critères d'évaluation du fonctionnement des peuplements cultivés et, enfin, de déterminer les régulations génétiques qui s'expriment à l'échelle de la plante individuelle et qui restent pertinentes à l'échelle de la population.

\section{REFERENCES}

1. SÉBILLOTTE M (1990). Système de culture, un concept opératoire pour les agronomes. In : COMBES L, PICARD D, éds. Un point sur... Les systèmes de culture. Paris : Inra Édition : 165-96.

2. COLÉNO FC, DURU M (1998). Gestion de production en systèmes d'élevage utilisateurs d'herbe : une approche par atelier. Etud Rech Syst Agraires Dév, 31 : 45-61.

3. LEMAIRE G, CHARRIER X, HÉBERT Y (1996). Nitrogen uptake capacities of maize and sorghum crops in different nitrogen and water supply conditions. Agronomie, $16: 231-46$.

4. BRISSON N, MARY B, RIPOCHE D, et al. (1998). STICS : a generic model for the simulation of crops and their water and nitrogen balances. Agronomie, $18: 311-47$.

5. JONES CA, KINIRY JR (1989). CERES-Maize, a simulation model of maize growth and development. College Station, Texas USA : Texas A\&M University Press.

6. LEMAIRE G, MEYNARD JM (1997). Use of the nitrogen nutrition index for the analysis of agronomical data. In : LEMAIRE G, ed. Diagnosis of the nitrogen status in crops. Heidelberg : Springer Verlag : 45-55. 